
T. SCHRÖDER ${ }^{1}$
C. LEMMERZ 1
O. REITEBUCH ${ }^{1, \infty}$
M. WIRTH ${ }^{1}$
C. WÜHRER ${ }^{2}$
R. TREICHEL ${ }^{3}$

\title{
Frequency jitter and spectral width of an injection-seeded Q-switched Nd:YAG laser for a Doppler wind lidar
}

\author{
${ }^{1}$ Institut für Physik der Atmosphäre, Deutsches Zentrum für Luft- und Raumfahrt (DLR) e.V., \\ 82234 Weßling, Germany \\ 2 EADS Astrium GmbH, Earth Observation \& Science, Ludwig-Bölkow-Allee, 85821 Ottobrunn, Germany \\ ${ }^{3}$ EADS Astrium GmbH, Earth Observation \& Science, 88039 Friedrichshafen, Germany
}

\section{Received: 6 June 2006/Revised version: 13 December 2006 Published online: 4 May 2007 • () Springer-Verlag 2007}

ABSTRACT The design of a $50 \mathrm{~Hz}$ single longitudinal mode, diode-pumped and frequency-tripled Nd:YAG master oscillator power amplifier is described, and the first measurements of output parameters are presented. The laser oscillator is injection-seeded by a tuneable monolithic Nd:YAG ring laser and frequency stabilized by minimising the Q-switch build-up time. The laser system will be an integral part of an airborne instrument demonstrator for a first satellite based Doppler wind lidar to measure vertical profiles of one component of the atmospheric wind vector. This paper focuses on the investigation of the frequency jitter and the linewidth of the laser, which are measured on a pulse-to-pulse basis. For this purpose a compact, high accuracy beat frequency monitoring system has been developed at DLR. By operating the amplifier stage at half the repetition rate $(50 \mathrm{~Hz})$ of the oscillator, we could reduce the frequency stability from $10 \mathrm{MHz}$ (rms) to $1.3 \mathrm{MHz}$ (rms) (over a $14 \mathrm{~s}$ period). We have determined a mean linewidth of $15 \mathrm{MHz}$ (FWHM) at $1064 \mathrm{~nm}$. These measured laser parameters enable wind velocity measurements in the atmosphere $(0-15 \mathrm{~km})$ at an accuracy of 1 to $2 \mathrm{~m} / \mathrm{s}$.

PACS 42.55.Xi; 42.60.Lh; 42.68.Wt

1

\section{Introduction}

The measurement of atmospheric wind profiles from ground and airborne platforms is a basic requirement in meteorology. Particularly, reliable instantaneous global analyses of winds are needed to improve the quality of the numerical weather prediction, and also to improve the understanding of atmospheric dynamics and climate processes [1]. Initiated by the European Space Agency ESA currently a direct detection Doppler lidar is being developed to sense global wind fields from a satellite for the first time [2].

In order to validate the performance of this Doppler lidar instrument, called ALADIN (Atmospheric Laser Doppler Instrument), and to obtain a dataset of atmospheric measurements a prototype for an airborne platform was developed. The ALADIN airborne demonstrator (A2D) is representative for the satellite based Doppler lidar. The A2D, designed to

Fax: +49-8153-28-1271, E-mail: oliver.reitebuch@dlr.de fly on the Falcon 20 aircraft from DLR, operates as a direct detection Doppler wind lidar at $355 \mathrm{~nm}$ wavelength retrieving aerosol and molecular backscatter signals in parallel. A Fabry-Pérot interferometer is used to measure the Doppler shift of the spectrally broadened molecular signal while a Fizeau interferometer is used for the narrowband aerosol return. The main task of the A2D is the measurement of the line-of-sight (LOS) component of the wind vector in the atmosphere $(0-15 \mathrm{~km})$ with a range resolution of $300 \mathrm{~m}$ to $1.2 \mathrm{~km}$. The projection of the line-of-sight onto the horizontal (HLOS) should be measured with an accuracy of 1 to $2 \mathrm{~m} / \mathrm{s}$ depending on range. Results of the development, the manufacturing of the A2D, and the campaign objectives have been presented elsewhere [3,4].

The laser transmitter - as key component of the Doppler lidar system - must comply with constraints for airborne applications such as compactness, ruggedness and low power consumption. Pulse energy, pulse length, repetition rate, spatial and spectral beam properties are essential laser parameters which determine the instrumental performance of the lidar system. One of the most challenging goals in the development of the laser transmitter is to achieve a frequency stability which should be in the order of several $\mathrm{MHz}(\mathrm{rms})$ at medium output energies of about $70 \mathrm{~mJ} /$ pulse at $355 \mathrm{~nm}$. In order to reach the measurement goals of the A2D, the laser transmitter is designed as a single mode, $70 \mathrm{~mJ}, 50 \mathrm{~Hz}$ pulse repetition rate, diode-pumped and frequency tripled Nd:YAG laser in a master oscillator power amplifier (MOPA) configuration. Stable single-mode operation and tuning is achieved by injection seeding the oscillator in combination with the Q-switch build-up time (QBUT) minimisation technique [5, 6] to match the axial mode frequency of the slave laser to the seeder frequency. Medium power single-mode Nd:YAG laser systems nearest to the optical specifications for the A2D are the systems reported by Ehret et al. [7] using the QBUT technique and Ostermeyer et al. [8], applying a modified Pound-DreverHall scheme. The latter was claimed to achieve a frequency stability of better than a few MHz at $1064 \mathrm{~nm}$, but no measurements were presented.

The knowledge of the frequency stability and spectral width of the laser and the compliance during the wavelength calibration of the receiver as well as during the wind measurements requires the deployment of an appropriate monitoring system. The optical heterodyning detection method, 
which is used in coherent Doppler lidar systems [9] for measuring the Doppler-shift allows the recording of the shot-toshot frequency jitter of a pulsed laser with high accuracy. By some authors the optical heterodyning method was applied for studying the instantaneous frequency fluctuations of nanosecond single mode laser pulses for precision laser spectroscopy [10-13]. Compared to methods based on a spectrum analyzer or a Fabry-Pérot etalon [14] the spectrum of individual pulses can be determined, as opposed to the average spectrum of an ensemble of pulses.

This paper is organized as follows. Section 2 outlines essential optical requirements such as pulse energy, frequency stability and linewidth and describes in detail the optical layout of the A2D laser transmitter. In Sect. 3 we describe the design of the optical heterodyne measuring system. Section 4 presents output parameters and measurements of the frequency fluctuations and the linewidth of the laser transmitter on a pulse-to-pulse basis. The results are discussed related to the required characteristics of the airborne demonstrator. Finally, Sect. 5 summarizes the experimental results.

\section{$2 \quad$ Laser transmitter design}

2.1 Optical design

The optical design shown in Fig. 1 consists of the following main parts: a tuneable seed laser and a quasi fixed- frequency "reference laser", a low power oscillator (LPO), two amplifier stages, and a frequency conversion stage. The reference laser head was developed at Innolight GmbH, Germany; the frequency tripled Nd:YAG MOPA was designed at Thales Laser S.A., France. The LPO is injection seeded in order to achieve single longitudinal mode operation and frequency tuning. The amplification is achieved in two stages in order to reach the required pulse energy. Second and third harmonic generators (SHG, THG) convert the IR radiation to $532 \mathrm{~nm}$ and $355 \mathrm{~nm}$. The laser base plate is made of an iron-nickel alloy (Invar) which guaranties high thermomechanical stability. In order to cope with the spatial restrictions within the aircraft the laser head was constructed in a compact setup with dimensions of $344 \mathrm{~mm}$ (width) $\times$ $780 \mathrm{~mm}$ (length) $\times 352 \mathrm{~mm}$ (height). The optical heterodyne unit used for the laser frequency diagnostic gets the optical signals of the seed laser and of the residual pulsed IR laser beam via polarization-maintaining (PM) single mode fibres. The seed laser power is split up in a ratio of 50:50 in order to have a local oscillator signal for the heterodyne detection unit (see Sect. 3).

\subsection{Reference laser head}

The reference laser head (RLH) consists of two identical laser systems (reference laser and seed laser) and

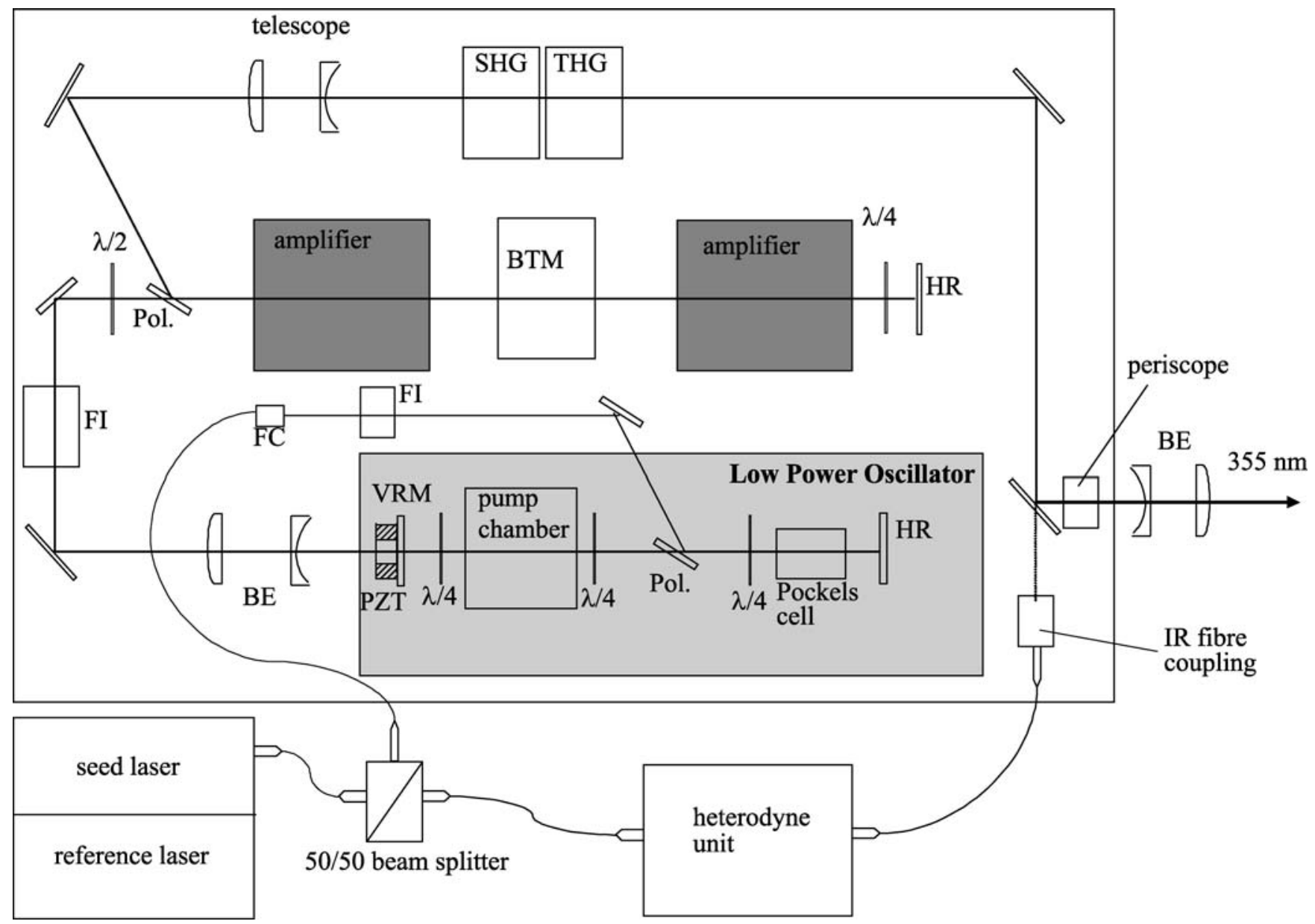

FIGURE 1 Schematic of the laser setup including the fibre coupling to the heterodyne unit 
a frequency stabilisation scheme. The reference laser - being the frequency reference - operates as a quasi fixed-frequency laser while the seed laser is continuously frequency tunable. The laser transmitter must be tuneable in frequency over the spectral range $\pm 5 \mathrm{GHz}$ of the two interferometers of the A2D receiver for wavelength calibration of the A2D. Reference and seed laser are both based on a laser diode pumped monolithic non-planar Nd:YAG ring oscillator with single longitudinal mode operation [15]. A phase-locked-loop controller is used in the RLH to hold and tune the seed laser at a defined difference frequency from the frequency stable reference laser. Frequency tuning of the seed laser is realized by a combination of a slow laser crystal temperature variation and a fast pump laser diode current modulation. The seed laser tunes mode-hop free over $15 \mathrm{GHz}$. The frequency stability of the reference laser was measured by heterodyning its reference radiation against an iodine stabilised Nd:YAG laser. The measured frequency stability was $234 \mathrm{kHz}(\mathrm{rms})$ over $25 \mathrm{~min}$ [16]. The maximum seed laser power was $90 \mathrm{~mW}$ measured at the fibre output.

\section{3}

\section{Low power oscillator (LPO)}

The pump chamber of the linear resonator is composed of an Nd:YAG rod transversally pumped by three standard diode stacks in a threefold radial symmetric configuration. Each stack provides a peak power of $350 \mathrm{~W}$ for $175 \mu \mathrm{s}$ pulse duration at $100 \mathrm{~Hz}$. The highly reflective mirror (HR) is concave with a reflectivity of $99 \%$. A convex Gaussian variable reflectivity mirror (VRM) is used as output coupler to ensure the required output beam parameters. With that configuration and a cavity length of about $30 \mathrm{~cm}$ pulse durations of more than $30 \mathrm{~ns}$ were obtained. To prevent the effect of spatial hole burning in the laser crystal, two quarter-wave-plates are placed in front of and after the pump chamber to have a circular polarisation within the rod (twisted mode technique). For the generation of actively controlled laser pulses a polarizer, a quarter-wave-plate and a Pockels cell as Q-switch are inserted. The output coupler mirror of the LPO is mounted on a piezoelectric translator (PZT) to allow control of the cavity length. The seed laser beam is mode matched to the oscillator cavity by means of a focusable fibre collimator (FC). The coupling occurs through a polarizer. A beam expanding telescope (BE) positioned behind the LPO fits the beam divergence and size for optimum power amplification. Two Faraday isolators (FI) protect the LPO and the seed laser from destabilizing feedback or actual damage from back-reflected light.

\section{4}

\section{Active frequency control}

The active frequency control of the LPO is achieved by injection seeding in combination with the QBUT minimisation technique. This is currently the most common method to ensure resonance of the slave laser cavity with the seed laser frequency. The principal relies on the fact, that the build-up time (time between actual pulse emission and Q-switch trigger) of the Q-switched pulses is shortest when a longitudinal mode of the oscillator corresponds to the seed laser frequency. By translating the output coupler by means of the PZT, the laser cavity is dithered by a small fraction of one free spectral range (FSR) of the resonator about the locked position

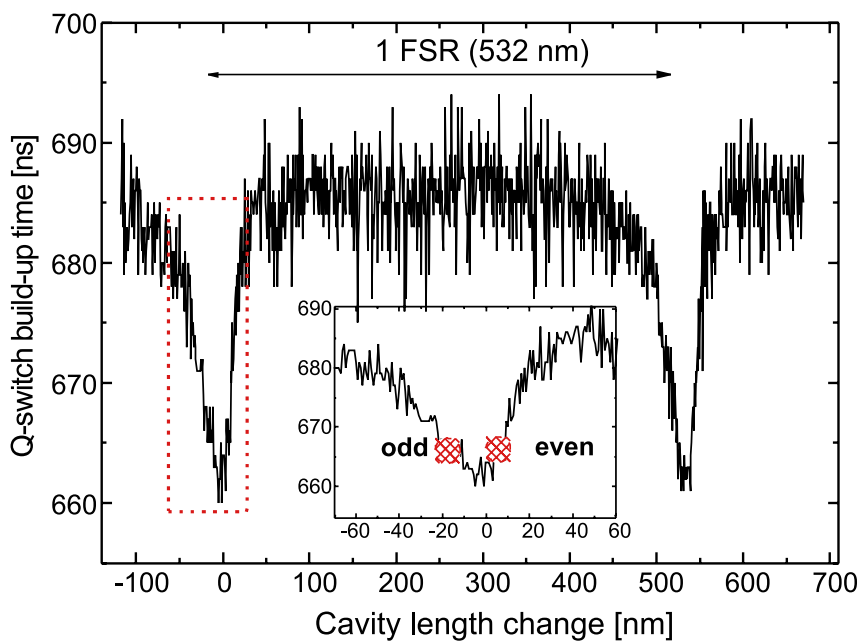

FIGURE 2 Measured Q-switch build-up time (including an offset of about $550 \mathrm{~ns}$ ) during scanning the PZT. The LPO is seeded with a power of about $40 \mathrm{~mW}$. The PZT dithers between two subsequent laser shots (odd and even) typically by about $5 \%$ of one FSR $(532 \mathrm{~nm})$. The measurement was performed using the data acquisition unit for the heterodyne detection unit (Sect. 3)

at $50 \mathrm{~Hz}$ rate. The difference in the build-up times of the two translator positions is fed back to correct the average translator position.

The measured Q-switch build-up time of the laser pulse vs. the cavity length change is shown in Fig. 2. If the oscillator cavity is in resonance with the seed laser frequency the Q-switch build-up time is minimised. On the first (odd) pulse the resonator is changed to a slightly shorter length from its average length, and then changed to a slightly longer length by the same amount on the subsequent (even) pulse. Because of this, the output frequency of the oscillator will alternate between two succeeding shots. The dither amplitude for achieving long term single frequency operation is typically about 5\% of the FSR [5]. According to the LPO's FSR of $400 \mathrm{MHz}$, this corresponds to an expected dither of the output frequency of $20 \mathrm{MHz}$. An alternation of the frequency between two subsequent shots by this amount is not tolerable compared with the requirement of $<1.3 \mathrm{MHz}$ (rms) at $1064 \mathrm{~nm}$. In order to considerably increase the shot-to-shot frequency stability the amplifier stages were operated at half of the oscillator repetition rate, i.e., at $50 \mathrm{~Hz}$, while the LPO operates at $100 \mathrm{~Hz}$.

\subsection{Amplifier stages}

A slab geometry has been selected as baseline for the two amplifier stages. This geometry allows effective removal of the strong thermal load deposited in the slab by the pump laser light. The Nd:YAG slab dimensions have been determined through optical and thermo-optical simulations to ensure an effective extraction. When double passed, eleven internal reflections have been determined as an optimum fill factor at given beam diameter and slab dimensions. Each slab is side-pumped by eight standard stacks $(1000 \mathrm{~W} /$ stack for about $185 \mu$ s pulse duration at $50 \mathrm{~Hz}$, synchronized on the oscillator). Between the two amplifier stages, three beam turning mirrors (BTM) rotate the beam spatially by $90^{\circ}$ after each pass 
for compensation of thermally induced astigmatism. After double pass amplification the beam is quasi-circular so that spherical optics can be used. A Galilean telescope in front of the harmonic generators reduces the spot size and adapts the divergence of the pump beam in order to optimize the UV conversion efficiency.

\section{6}

\section{Harmonic generators}

The nonlinear optical crystals for second and third harmonic generation are $\mathrm{LBO}\left(\mathrm{LiB}_{3} \mathrm{O}_{5}\right)$ crystals used in critical phase-matching orientation and temperature stabilized at $35^{\circ} \mathrm{C}$. The SHG crystal is $18 \mathrm{~mm}$ long, while the THG crystal is $16 \mathrm{~mm}$ long. The section is $7 \times 7 \mathrm{~mm}^{2}$ for both crystals. The high quality anti-reflection coatings at the entrance and exit faces of the SHG crystal (double band coating at $1064 \mathrm{~nm}$ and $532 \mathrm{~nm}$ ) and THG crystal (triple band coating at $1064 \mathrm{~nm}, 532 \mathrm{~nm}$ and $355 \mathrm{~nm}$ ) minimise the optical losses. Four heaters allow heating the mount symmetrically. A thermistor is attached to the mount as part of the temperature control circuit.

\section{Optical heterodyne method}

The spectral linewidth and the frequency jitter of the laser were measured by optical heterodyning the pulsed laser and the seed laser. Figure 3 illustrates the basic principle of the heterodyne detection. We consider two linearly polarized plane waves from the seed and from the pulsed laser which are mixed on a fast photodiode. The electric field from the seed laser is frequency shifted by the carrier frequency $\Omega_{\mathrm{AOM}}$ from an acousto-optic modulator (AOM). The electric fields are expressed as

$E_{\mathrm{ref}}(t)=A_{\mathrm{ref}} \exp \left[i \omega_{\mathrm{ref}} t\right]+c . c .$,

$E_{\mathrm{p}}(t)=A_{\mathrm{p}}(t) \exp \left[i \omega_{\mathrm{p}} t+i \Phi(t)\right]+c . c .$,

$A_{\text {ref: }}$ electric field amplitude of reference signal; $A_{\mathrm{p}}(t)$ : electric field amplitude of pulsed signal;

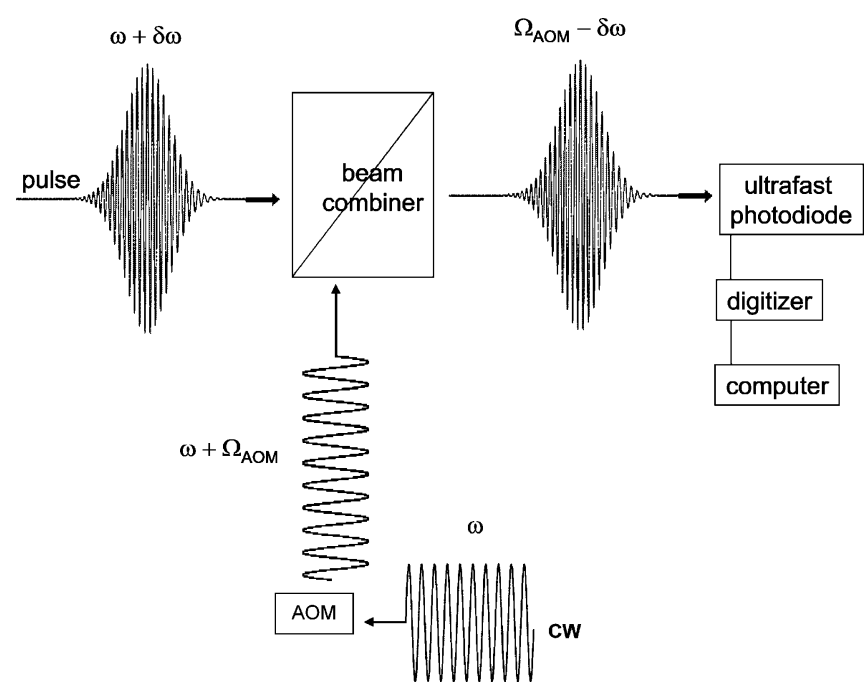

FIGURE 3 Schematic of the optical heterodyne method for measuring the frequency jitter and the linewidth on pulse-to-pulse basis $\omega_{\mathrm{p}}$ : centre frequency of pulsed laser;

$\omega_{\text {ref }}$ : frequency of seed laser shifted by $\Omega_{\mathrm{AOM}}$;

$\Phi(t)$ : phase perturbations of pulsed light relative to the reference field;

c.c.: complex conjugated.

The intensity of the detected signal $(I)$ is proportional to the product of the sum of the signals and their complex conjugate. The mixed signal contains the sum and the difference frequencies of the two components. The sum cannot be detected by the photodiode due to its limited bandwidth. But the difference is a low-frequency signal that can be determined with high accuracy. The time averaged detector signal results in

$\langle I\rangle \sim A_{\mathrm{ref}}^{2}+A_{\mathrm{p}}^{2}(t)+A_{\mathrm{p}}(t) A_{\mathrm{ref}} \cos \left[\left(\omega_{\mathrm{ref}}-\omega_{\mathrm{p}}\right) t+\Phi(t)\right]$.

The first term in (3) is the direct current (DC) component of the $\mathrm{cw}$ reference signal. The second term represents the intensity envelope of the optical pulse, and the third term is the optical heterodyne beat signal. The beat frequency $\omega_{\text {ref }}-\omega_{\mathrm{p}}=\Omega_{\mathrm{AOM}}-\delta \omega$ yields the deviation $\delta \omega$ between the frequencies of the seed laser and the pulsed laser. An analysis of the beat waveform by a fast-Fourier transform (FFT) technique provides the spectral intensity profile. From the derivation of the phase perturbations $\Phi(t)$ the frequency chirp of the optical pulse can be derived $[12,13]$. It should be emphasised that the determination of the absolute frequency drift and jitter of the pulsed laser requires the heterodyning against a more frequency stable source such as a iodine stabilized Nd:YAG laser.

The developed heterodyne detection unit completely uses fibre optics which greatly simplifies measurements and solves many optical alignment problems. For maintaining the polarisation state of the optical fields we used PM single mode fibres for beam guiding. A fraction of the seed laser beam (about $10 \mathrm{~mW}$ ) is used as reference input for the heterodyne measurement. The optical pulse, whose frequency spectrum has to be analysed, is attenuated by the leakage of the two $45^{\circ}$ dichroic mirrors within the power laser head and an adjustable threaded radial screw located at the input port of the beam combiner within the heterodyne detection unit. The peak power of the pulsed optical signal was set to a level comparable to the reference signal. The seed laser frequency is shifted by $200 \mathrm{MHz}$ using a fibre pigtailed AOM. A $1: 1$ PM beam combiner combines both optical signals which are mixed by a $3.5 \mathrm{GHz}$-bandwidth InGaAs PIN photodiode followed by an amplifier. The output of the photodetector amplifier is digitized by a high speed digitizer card at a sampling rate of 2 GSamples/s. The resulting digitized signal is transferred to a computer for processing. A FFT algorithm using the Hanning window type provides the spectral intensity of the seeded laser pulse. The centre beat frequency is given by the centre of gravity of the beat spectrum considering the fourfold spectral bandwidth around the maximum. The AOM used as frequency shifter is driven by a $200 \mathrm{MHz}$ signal generated by a quartz oscillator having $5 \times 10^{-5}$ frequency stability. As a result, the systematic error of a beat frequency measurement is $<10 \mathrm{kHz}$.

A typical heterodyne beat signal - recorded and digitized by a $1 \mathrm{GHz}$ analogue bandwidth oscilloscope at $16 \mathrm{GSam}-$ ples/s - is shown in Fig. 4. A FFT of this signal leads to the 


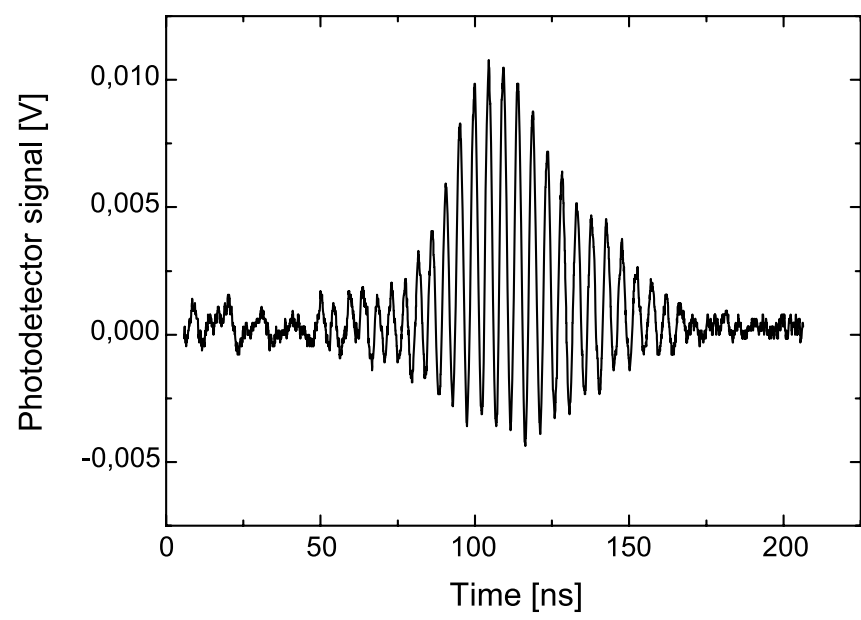

FIGURE 4 Heterodyne beat signal of a typical laser pulse with injection seeding at $1064 \mathrm{~nm}$ recorded by a $1 \mathrm{GHz}$ analogue bandwidth oscilloscope at 16 GSamples/s sampling rate

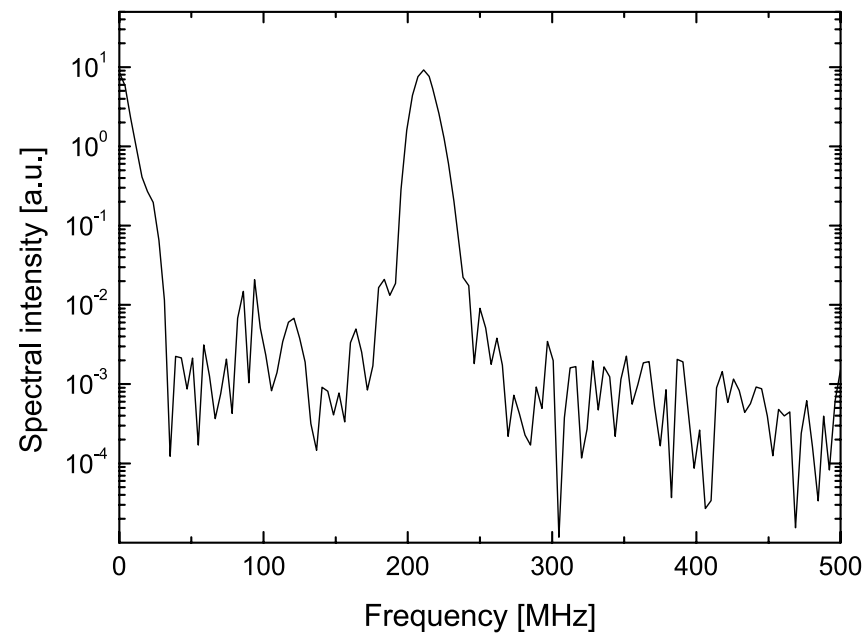

FIGURE 5 Fast-Fourier transform of a single heterodyne beat signal, showing the positive portion of the power spectrum. The spectrum represents the intensity term at $0 \mathrm{~Hz}$ and the interference term at $210 \mathrm{MHz}$

corresponding power spectrum shown in Fig. 5. The peak at $0 \mathrm{~Hz}$ is associated with the pulse intensity term $A_{\mathrm{p}}^{2}(t)$ of (3), and the peak at $210 \mathrm{MHz}$ is associated with the interference term. The measured signal frequencies are below the Nyquist frequency of $8 \mathrm{GHz}$ ensuring alias-free sampling. The offset of the centre beat frequency compared to the $200 \mathrm{MHz}$ frequency shift of the seed frequency depends on the PZT dither amplitude.

\section{$4 \quad$ Measured laser parameters}

The master oscillator provides a pulse with nearly diffraction limited beam profile and an output energy of $10 \mathrm{~mJ}$ at $100 \mathrm{~Hz}$ pulse repetition rate. The measured IR pulse energy after double pass amplification exceeds $200 \mathrm{~mJ}$ (Fig. 6) at $50 \mathrm{~Hz}$ repetition rate. Without injection seeding the pulse shape shows strong modulations due to mode beating (Fig. 7). With injection seeding the mode beating is suppressed and the pulses show a smooth shape as expected for single mode operation. The measured FWHM pulse duration at $1064 \mathrm{~nm}$ was 35 ns. After frequency tripling the pulse length was reduced

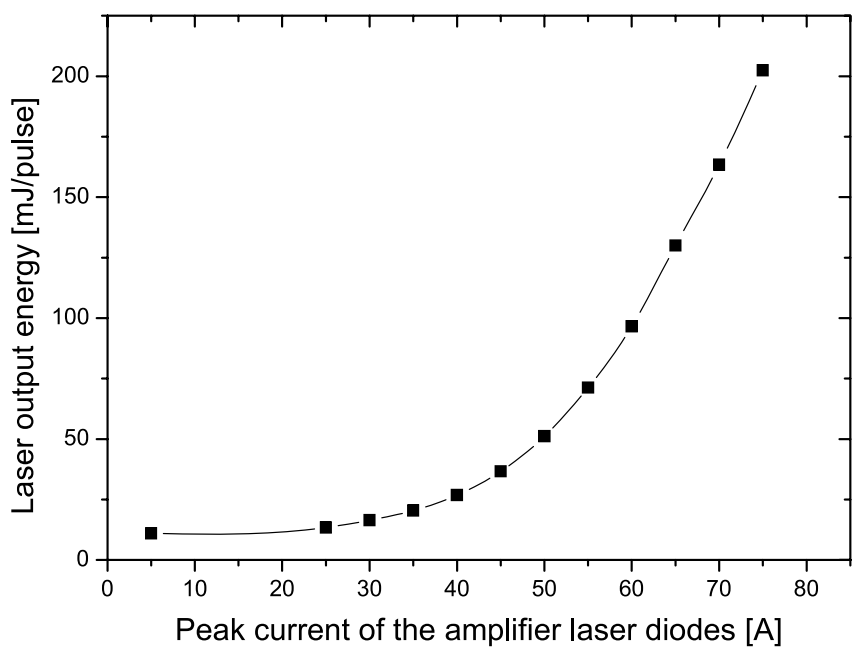

FIGURE 6 IR output energy of the injection seeded Nd:YAG laser vs. peak current of the amplifier pump diodes

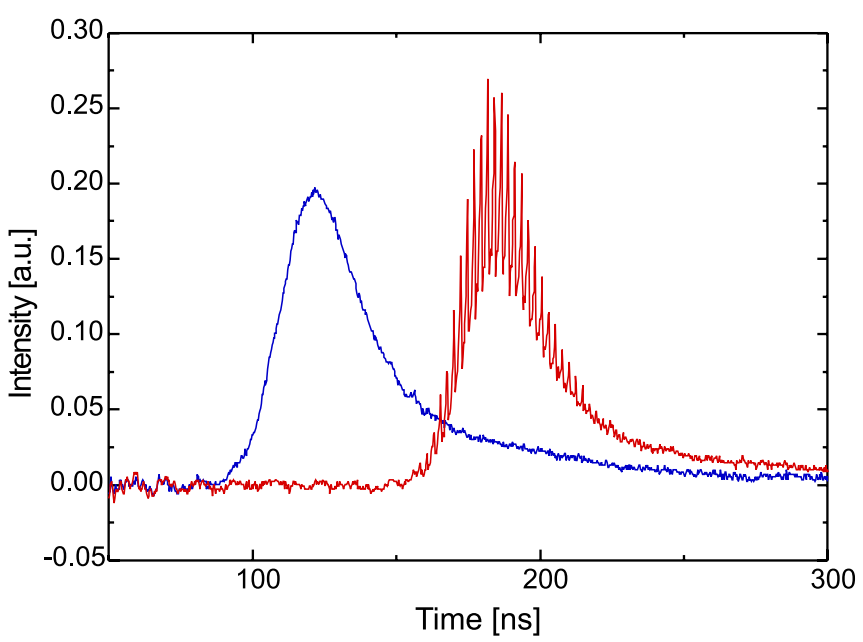

FIGURE 7 Temporal pulse shapes of the laser transmitter at $1064 \mathrm{~nm}$ in seeded and unseeded operation. When the laser is seeded its Q-switch buildup time was reduced by $60 \mathrm{~ns}$. The pulse shape was measured with a $2 \mathrm{GHz}$ InGaAs photodiode and a $1 \mathrm{GHz}$ analogue bandwidth oscilloscope. The IR pulse duration (FWHM) is $35 \mathrm{~ns}$ at $200 \mathrm{~mJ} /$ pulse output energy

to $25 \mathrm{~ns}$. The UV conversion efficiency was measured to be $30 \%$ which corresponds to $E=60 \mathrm{~mJ} /$ pulse output energy at $355 \mathrm{~nm}$

As has been shown in simulations of the A2D performance [17] the maximum expected wind speed random error is $<0.9 \mathrm{~m} / \mathrm{s}$ (700 accumulated laser pulses) for $E=$ $60 \mathrm{~mJ} /$ pulse for an airborne system at $12 \mathrm{~km}$ flight altitude. This value is still below the envisaged wind speed measurement accuracy of $1-2 \mathrm{~m} / \mathrm{s}$ (HLOS).

Table 1 lists the output performance values which have been measured including frequency stability and linewidth which are described in the following subsections.

\section{1}

\section{Frequency stability}

The analysis of the frequency jitter refers to a recording interval of $14 \mathrm{~s}$ (700 shots) which is the averaging time of the ALADIN lidar receiver for one line-of-sight wind profile measurement. The Q-switch build-up time min- 


\begin{tabular}{lcc}
\hline Parameter & at $1064 \mathrm{~nm}$ & at $355 \mathrm{~nm}$ \\
\hline $\begin{array}{l}\text { Pulse energy of oscillator } \\
\text { Pulse energy with amplification }\end{array}$ & $10 \mathrm{~mJ}(100 \mathrm{~Hz})$ & \\
(max. values) & $200 \mathrm{~mJ}$ & $60 \mathrm{~mJ}$ \\
$\begin{array}{l}\text { Pulse repetition rate } \\
\text { Polarization }\end{array}$ & $50 \mathrm{~Hz}$ & $50 \mathrm{~Hz}$ \\
Pulse duration (FWHM) & linear, purity $>100: 1$ & \\
Frequency stability (over 14 s) & $35 \mathrm{~ns}$ & $25 \mathrm{~ns}$ \\
Linewidth (FWHM) & $1.3 \mathrm{MHz}(\mathrm{rms})$ & \\
\hline
\end{tabular}

TABLE 1 Measured output parameters of the Nd:YAG MOPA laser
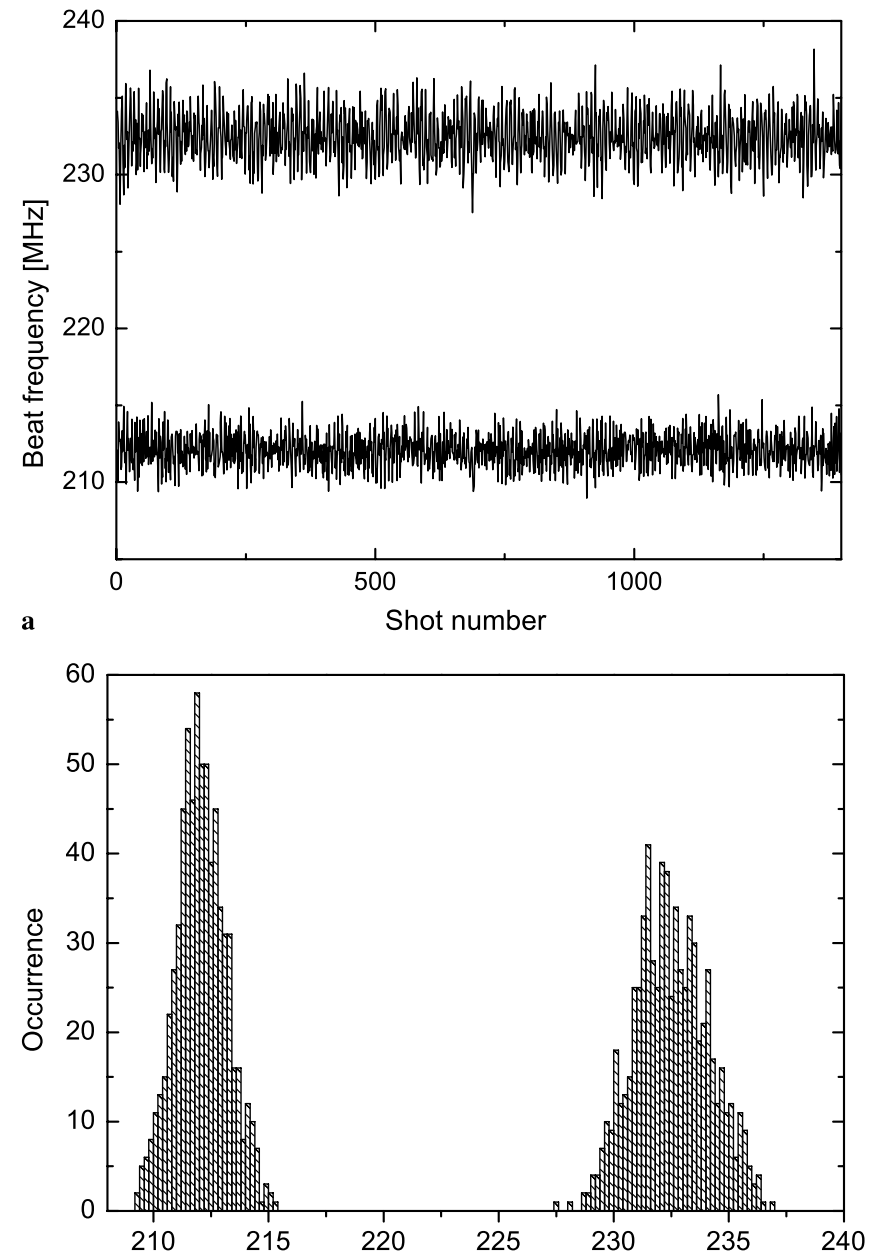

b

FIGURE 8 Frequency stability measured over $14 \mathrm{~s}$ with the amplifiers operating at $100 \mathrm{~Hz}$ repetition rate. (a) Time behaviour of the short-term frequency fluctuations recorded shot-to-shot over $14 \mathrm{~s}$. The frequencies of odd and even shots differ by $20 \mathrm{MHz}$. (b) Histogram of the measured central frequency distribution with a bin size of $0.2 \mathrm{MHz}$. The frequency jitter is $1.1 \mathrm{MHz}(\mathrm{rms})$ for the odd and $1.6 \mathrm{MHz}$ for the even shots

imization technique leads to an output pulse frequency at two alternating mean values with a distance of typically tens of $\mathrm{MHz}$ as confirmed from the measurements displayed in Fig. 8a and b. From that measurement the standard deviation of the frequency stability at $1064 \mathrm{~nm}$ is $10.3 \mathrm{MHz}$. In order to further restrict the frequency jitter, the amplifiers were operated at half the repetition rate $(50 \mathrm{~Hz})$ while the oscillator was pumped at $100 \mathrm{~Hz}$. By this means the
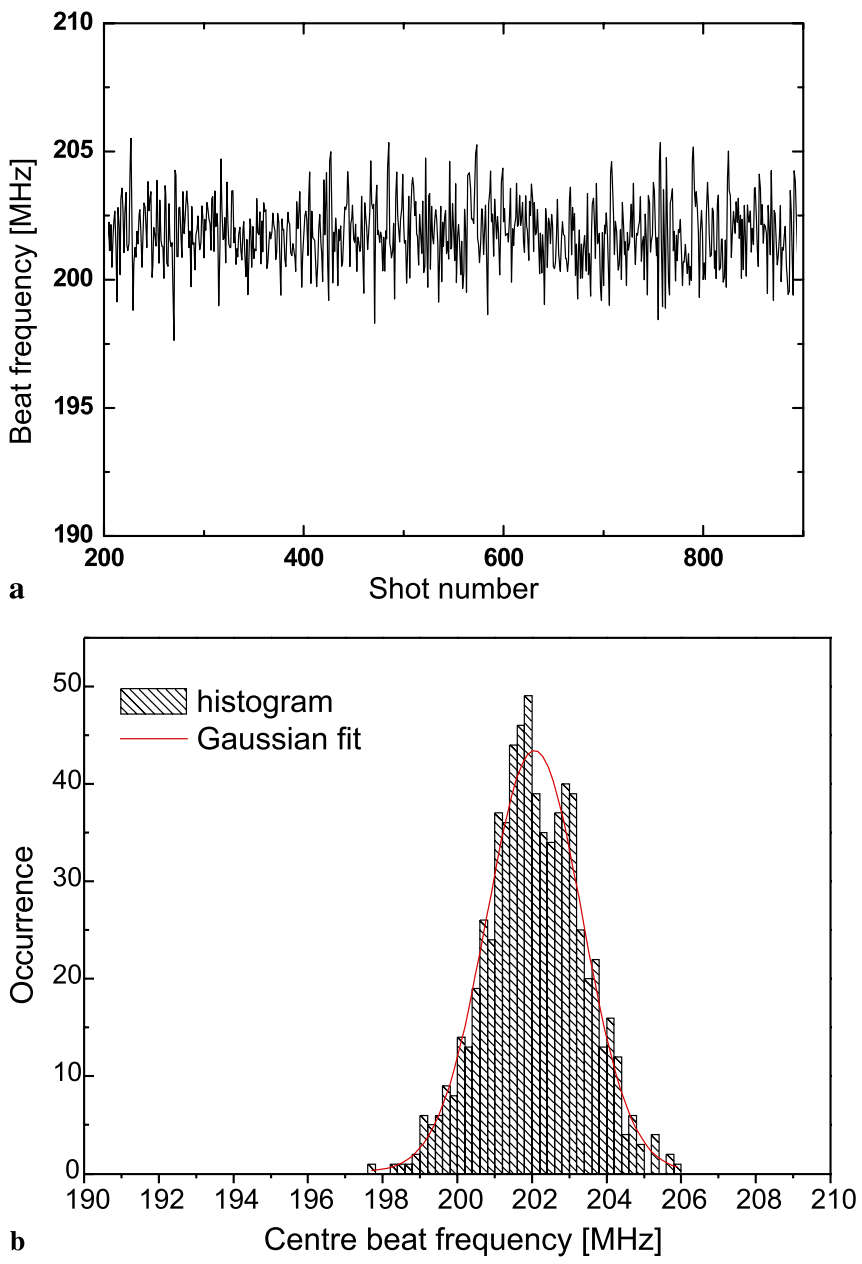

FIGURE 9 Frequency stability measured over $14 \mathrm{~s}$ with the amplifiers operating at $50 \mathrm{~Hz}$ repetition rate which avoids alternating between two frequencies. (a) Time behaviour of the short-term frequency fluctuations recorded shot-to-shot over $14 \mathrm{~s}$. (b) Histogram of the measured central frequency distribution with a bin size of $0.2 \mathrm{MHz}$. The solid line represents the Gaussian distribution which fits best to the data. The mean centre beat frequency is $202 \mathrm{MHz}$; the frequency stability for this observation period is $1.3 \mathrm{MHz}(\mathrm{rms})$

frequency jitter was limited to $1.3 \mathrm{MHz}(\mathrm{rms})$ over $14 \mathrm{~s} \mathrm{ob-}$ servation time (see Fig. 9a, b). This value corresponds to a relative laser frequency stability of $5 \times 10^{-9}$ over this period. The seed laser frequency fluctuates with a stability of $234 \mathrm{kHz}(\mathrm{rms})$ measured over $25 \mathrm{~min}$ providing the lower limit for the frequency stability of the laser transmitter. Figure $9 \mathrm{~b}$ shows the distribution of the measured centre beat frequencies. The histogram fits well to a Gaussian distribution demonstrating that the laser frequency fluctuates statistically independent.

The error of a wind velocity measurement with A2D increases with shot-to-shot frequency fluctuations of the laser transmitter. For example, the aerosol backscatter signal at $355 \mathrm{~nm}$ is Doppler shifted w.r.t. the transmitted laser pulse by $5.6 \mathrm{MHz}$ for each $\mathrm{m} / \mathrm{s}$ wind speed in LOS direction. The frequency stability of the laser transmitter at $355 \mathrm{~nm}$ was set to be below $4 \mathrm{MHz}$ (rms) [4]. By transforming the measured frequency jitter of $1.3 \mathrm{MHz}(\mathrm{rms})$ from $1064 \mathrm{~nm}$ to $355 \mathrm{~nm}$ the corresponding value at $355 \mathrm{~nm}$ is $3.9 \mathrm{MHz}$ (rms) which meets the requirement of $<4 \mathrm{MHz}$ (rms). 


\section{2}

\section{Linewidth}

The linewidth of the pulsed laser at $1064 \mathrm{~nm}$ is derived by a FFT of the heterodyne beat signal. Figure 10 displays the shot-to-shot FWHM linewidths measured by means of the heterodyne detection unit over a period of $14 \mathrm{~s}$. The mean value is $14.8 \mathrm{MHz}$ with a standard deviation of $1.3 \mathrm{MHz}$, while the laser operated single frequency at about $200 \mathrm{~mJ}$ output pulse energy $(1064 \mathrm{~nm})$.

In order to guarantee the wind measurement accuracy the laser linewidth must be appreciably lower than the instrumental resolution of the A2D receiver. The determined resolution for the aerosol Fizeau spectrometer is $135 \mathrm{MHz}$ at $355 \mathrm{~nm}$ [18]. The limit for the mean laser linewidth for the ALADIN receiver is $50 \mathrm{MHz}$ at $355 \mathrm{~nm}$ [4] which corresponds to $17 \mathrm{MHz}$ at $1064 \mathrm{~nm}$ as upper limit. As seen from Fig. 10, the measured mean linewidth of $14.8 \mathrm{MHz}$ meets the requirement for the $\mathrm{A} 2 \mathrm{D}$ receiver.

A more detailed characterization of the spectral distribution of the laser output is given by the determination of its Fourier transform limit. It is derived by a FFT of the field envelope $A_{\mathrm{p}}(t)$ (2) providing the Fourier transform limited intensity spectrum $I_{\mathrm{FTL}}(v)$. In order to get $A_{\mathrm{p}}(t)$, we fitted the measured intensity profile (see Fig. 11) by the fit function $f(t)=[\exp (t / \tau)+\exp (-3 t / \tau)]^{-2}$ with $\tau=33 \mathrm{~ns}$ considering a fast rise time and a slow fall time. The square root of this fit function provides $A_{\mathrm{p}}(t)$ which was Fourier transformed to get $I_{\mathrm{FTL}}(v)$ (see Fig. 12). As a result, the effective laser linewidth is a factor of two above the Fourier-transform-limit.

The intensity spectrum of the Nd:YAG MOPA is spectrally broadened by a frequency chirp which can be caused by the population inversion change during the pulse evolution [19]. From preliminary heterodyning measurements followed by an analysis of the instantaneous frequency within the pulse we have estimated an overall frequency chirp of less than $15 \mathrm{MHz}$. We expect a negligible influence of a laser frequency chirp in the A2D wind measurement because the vertical resolution of the A2D instrument is $2.1 \mu \mathrm{s}$, which is two orders

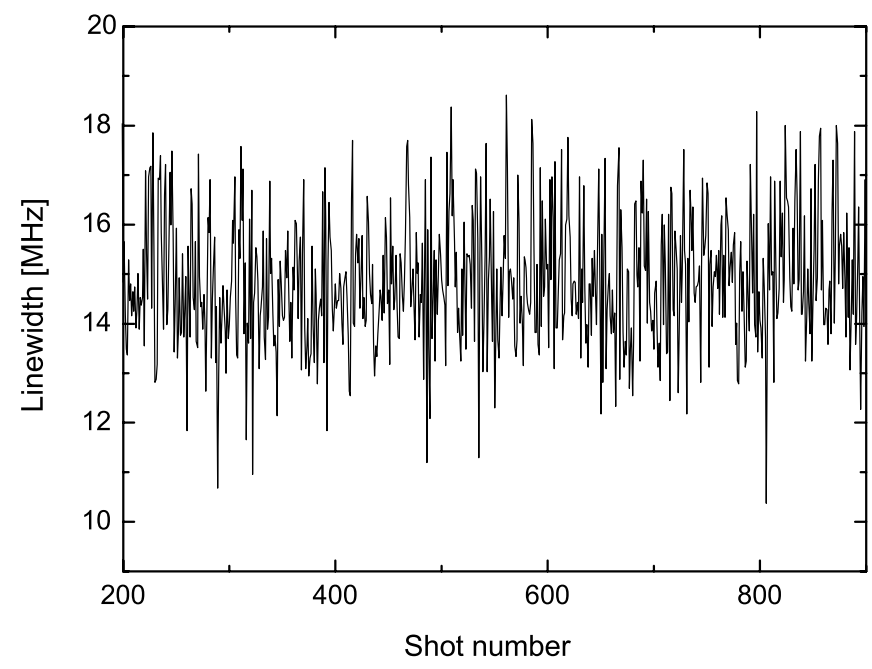

FIGURE 10 Laser linewidths on pulse-to-pulse basis measured with the optical heterodyne detection unit. The linewidth at FWHM is $14.8 \pm 1.3 \mathrm{MHz}(\mathrm{rms})$ measured over $14 \mathrm{~s}$

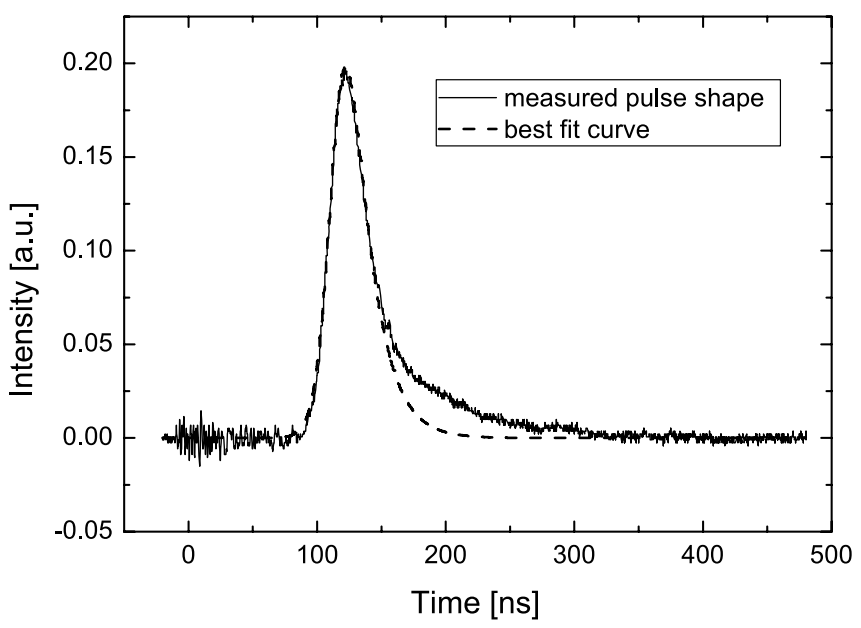

FIGURE 11 Single temporal pulse shape $I(t)$ of the laser at $1064 \mathrm{~nm}$ with injection seeding. The FWHM pulse duration of the measured temporal pulse $I(t)$ (solid line) is $35 \mathrm{~ns}$. The dashed line represents the best fit curve

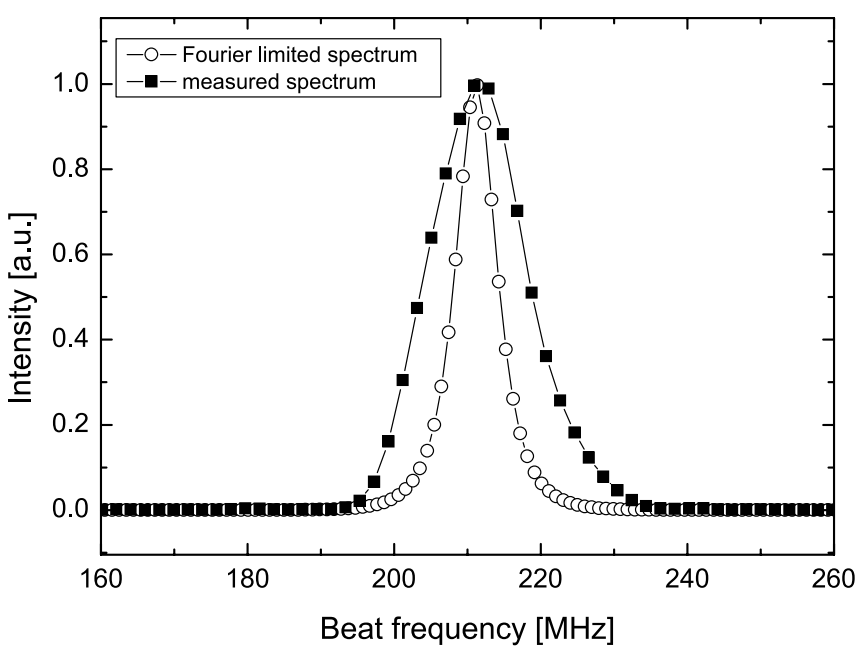

FIGURE 12 Comparison of the measured intensity spectrum I(v) (squares) (see Fig. 5) with the Fourier-transform-limited spectrum $I_{\text {FTL }}(v)$ (circles) which is derived by a FFT of the square root of the curve fit to the measured temporal shape $I(t)$ shown in Fig. 11. Both Fourier transformations were performed with a Hanning window. As a result the measured linewidth of the $\mathrm{Nd}$ :YAG MOPA laser is a factor of 2 above the Fourier-transform-limit

of magnitudes higher than the $25 \mathrm{~ns}$ duration of the UV laser pulse.

\section{$5 \quad$ Summary and conclusions}

We investigated the short-term (14s) frequency fluctuations and the linewidth of an injection seeded single longitudinal mode Nd:YAG laser, which is frequency stabilized by the Q-switch build-up time minimisation method. Furthermore, we presented the optical layout and first optical output parameters of the Nd:YAG MOPA laser system which is being developed for a lidar transmitter in an airborne direct detection Doppler lidar.

The diode-pumped injection seeded laser, built up in a MOPA design, is frequency-tripled and emits $25 \mathrm{~ns}$ long single mode laser pulses with a repetition rate of $50 \mathrm{~Hz}$. The output energy after the conversion stage is $>60 \mathrm{~mJ}$ (at $355 \mathrm{~nm}$ ) corresponding to a conversion efficiency of $30 \%$ 
from $1064 \mathrm{~nm}$ to $355 \mathrm{~nm}$. In order to characterise the spectral fluctuations of the laser we developed a rugged and compact instrument tool basing on the optical heterodyning technique. The instrument uses fibre optics to simplify the measurement and to make it suitable for airborne applications. The heterodyne detection unit acquires and processes the heterodyne beat signals on a pulse-to-pulse basis up to $500 \mathrm{~Hz}$ pulse repetition rate, and measures the frequency jitter of the pulsed laser with a systematic error of $<10 \mathrm{KHz}$. The frequency jitter and relative frequency drift of the pulsed laser against the seed laser will be measured routinely during wavelength calibration of the Fabry-Pérot and Fizeau interferometer of the receiver and during wind velocity measurements. The knowledge of these data will finally improve the accuracy of the Doppler lidar wind measurements.

The measured laser frequency stability on short-term basis (14 s) was $10 \mathrm{MHz}(\mathrm{rms})$ at $100 \mathrm{~Hz}$ repetition rate. By selecting every second shot for amplification the frequency stability of the laser could be reduced to $1.3 \mathrm{MHz}$ (rms) at $1064 \mathrm{~nm}$ according to a relative laser frequency stability of $5 \times 10^{-9}$ over this period. The measured linewidth of the pulsed laser is $14.8 \pm 1.3 \mathrm{MHz}$ (over $14 \mathrm{~s}$ ) at $1064 \mathrm{~nm}$. We have shown that the Fourier-transform-limit of the linewidth is exceeded by a factor of two, which is due to a frequency chirp. In summary, all investigated laser parameters cope with the lidar system requirements for measuring the horizontal line-of-sight component of the wind vector in the atmosphere $(0-15 \mathrm{~km})$ with a spatial resolution of $250 \mathrm{~m}$ to $1 \mathrm{~km}$ and an accuracy of 1 to $2 \mathrm{~m} / \mathrm{s}$.

Our measurements demonstrated that the common build up time method is sufficient to meet the requirements on frequency stability for an operational Doppler wind lidar on ground. Other relevant laser parameters will be characterized including beam pointing stability, beam divergence, longterm frequency stability and drift which influence the performance of the Doppler lidar system. In a further step the laser transmitter setup will be adapted to the operation on board of the DLR Falcon 20 aircraft.

ACKNOWLEDGEMENTS The experiments were carried out within the scope of the Atmospheric Dynamics Mission (ADM-Aeolus) Campaigns project financially supported by the European Space Agency ESA, Noordwijk, the Netherlands and DLR. The development of the A2D laser transmitter was funded by the pre-development programme of ESA. Special thanks go to M. Hunnekuhl, Innolight $\mathrm{GmbH}$, Germany for providing an iodine stabilised Nd:YAG laser and supporting the frequency stability measurements of the reference laser. The authors would like to thank, S. Rahm and R. Simmet for technical support and allocation of the data acquisition unit, and G. Ehret for providing fruitful comments to the manuscript.

\section{REFERENCES}

1 A. Stoffelen, J. Pailleux, E. Källén, J.M. Vaughan, L. Isaksen, P. Flamant, W. Wergen, E. Andersson, H. Schyberg, A. Culoma, R. Meynart, M. Endemann, P. Ingmann, Bull. Am. Metereol. Soc. 86, 73 (2005)

2 M. Endemann, P. Dubock, P. Ingmann, R. Wimmer, D. Morancais, D. Demuth, The ADM-Aelous Mission - The First Wind-Lidar in Space in Reviewed and Revised Papers Presented at the 22nd International Laser Radar Conference, ILRC 2004, Matera, Italy, ed. by G. Pappalardo, A. Amodeo (ESA Publication Division, ESTEC, Noordwijk, The Netherlands, 2004), 953

3 O. Reitebuch, E. Chinal, Y. Durand, M. Endemann, R. Meynart, D. Morancais, U. Paffrath, Development of an Airborne Demonstrator for ADM-Aeolus and Campaign Activities in Reviewed and Revised Papers Presented at the 22nd International Laser Radar Conference, ILRC 2004, Matera, Italy, ed. by G. Pappalardo, A. Amodeo (ESA SP-561, Paris: European Space Agency, 2004), 1007

4 Y. Durand, R. Meynart, M. Endemann, E. Chinal, D. Morancais, T. Schröder, O. Reitebuch, Proc. SPIE 5984 (2005)

5 L.A. Rahn, Appl. Opt. 24, 940 (1985)

6 R.L. Schmitt, L.A. Rahn, Appl. Opt. 25, 629 (1986)

7 G. Ehret, H.H. Klingenberg, U. Hefter, A. Assion, A. Fix, G. Poberaj, S. Berger, S. Geiger, Q. Lü, Laser Optoelectron. 32, 29 (2000)

8 M. Ostermeyer, P. Kappe, R. Menzel, V. Wulfmeyer, Appl. Opt. 44, 582 (2005)

9 C. Werner, Doppler Wind Lidar, in Lidar Range-Resolved Optical Remote Sensing of the Atmosphere, ed. by C. Weitkamp, in Springer Series in Optical Sciences, Springer, New York, 2005, pp. 325-354

10 M.S. Fee, K. Danzmann, S. Chu, Phys. Rev. A 45, 4911 (1992)

11 S. Gangopadhyay, N. Melikechi, E.E. Eyler, J. Opt. Soc. Am. B 11, 231 (1994)

12 R.T. White, Y. He, B.J. Orr, M. Kono, K.G.H. Baldwin, J. Opt. Soc. Am. B 21, 1577 (2004)

13 R.T. White, Y. He, B.J. Orr, M. Kono, K.G.H. Baldwin, J. Opt. Soc. Am. B 21, 1586 (2004)

14 J. Guena, P. Jacquier, L. Pottier, Meas. Sci. Technol. 1, 1254 (1990)

15 T.J. Kane, R.L. Byer, Opt. Lett. 10, 65 (1985)

16 M. Hunnekuhl, Innolight $\mathrm{GmbH}$, Germany, personal communication

17 U. Paffrath, Performance Assessment of the Aeolus Doppler Wind Lidar Prototype, ISSN 1434-8454, DLR-Forschungsbericht 2006-12 (2006)

18 M. Schillinger, D. Morancais, F. Fabre, A. Culoma, Proc. SPIE 4881, 40 (2003)

19 P. Bakule, P.E.G. Baird, M.G. Boshier, S.L. Cornish, D.F. Heller, K. Jungmann, I.C. Lane, V. Meyer, P.H.G. Sandars, W.T. Toner, M. Towrie, J.C. Walling, Appl. Phys. B 71, 11 (2000) 